\title{
RESPUESTA DE Bostryx conspersus Y Succinea peruviana (MOLLUSCA, GASTROPODA) AL EVENTO "EL NIÑO 1997-98" EN LAS LOMAS DE LACHAY (LIMA, PERÚ)
}

\author{
RESPONSE OF Bostryx conspersus AND Succinea peruviana \\ (MOLLUSCA, GASTROPODA) TO THE "EL NIÑO 1997-98" EVENT \\ IN THE LOMAS OF LACHAY (LIMA, PERÚ)
}

\author{
Rina Ramírez, Katia Caro, Saida Córdova, Janine Duárez, Asunción Cano, \\ César Arana y José Roque*
}

\section{RESUMEN}

Se presenta un análisis de las respuestas de dos caracoles terrestres, Bostryx conspersus (Bulimulidae) y Succinea peruviana (Succineidae) de las Lomas de Lachay (Lima, Perú) a las condiciones ambientales cambiantes antes, durante y después del evento "El Niño 1997-98". Ambas respondieron con la misma tendencia a los dos picos de "El Niño 1997-98", retardando el inicio y recortando sus periodos de actividad en 1997, y no entraron en estivación en el verano de 1998, más bien con una explosión poblacional. Por otro lado, es diferencial la intensidad de las respuestas. Estos cambios se relacionaron positivamente con otro factor cambiante durante "El Niño 1997-98", la cobertura vegetal.

Palabras claves: Bostryx conspersus, Succinea peruviana, Mollusca, El Niño, Lachay.

\section{ABSTRACT}

The analysis of the responses of two land snail species, Bostryx conspersus (Bulimulidae) and Succinea peruviana (Succineidae) from the Lomas of Lachay (Lima, Peru) to the changing environmental conditions before, during and after "EI Niño 1997-98" event is presented herein. Both species showed a remarkable response to the two picks of "El Niño 1997-98". During 1997, the start of the activity period of both species was delayed, while in the summer of 1998 neither $B$. conspersus nor $S$. peruviana had a period of aestivation at all, on the contrary, the populations of both species had a demographic explosion. However, the intensity of the response of those species to "El Niño" was not the same. These changes in land snails population are positively related with other factor, the vegetable cover, changeable during " $\mathrm{EI}$ Niño 1997-98".

Key words: Bostryx conspersus, Succinea peruviana, Mollusca, EI Niño, Lachay.

\section{INTRODUCCIÓN}

Los eventos "El Niño" representan la interacción dinámica de los océanos con el resto de la tierra (Bayona, 1985; Arntz y Fahrbach, 1991). Sus efectos biológicos en el ambiente marino del Perú están bien documentados no así en el continente (Arntz et al., 1985; Vega, 1985; Arntz y Fahrbach,

\footnotetext{
* Museo de Historia Natural - Univ. Nac. Mayor de San Marcos. A.P. 14-0434, Lima-14, Perú
}

1991), cuyo desierto costero se ve afectado por las inusuales lluvias (Bayona, 1985; Aguilar, 1990). Las lomas, islas de vegetación en el desierto, son ecosistemas estacionales, por lo que la flora y la fauna que componen su biota están adaptadas a las condiciones climáticas desfavorables durante el verano (Aguilar, 1985; Rundel et al., 1991). Los cambios medioambientales producidos por el evento "El Niño" modifican la fisonomía de las lomas, como consecuencia de la abundante humedad y precipitación (Torres, 1985; 
Pinche, 1994), y se espera que también produzcan cambios en las poblaciones de plantas y animales, los cuales pueden apreciarse haciendo el seguimiento en una serie de tiempo, tanto mejor cuanto más larga sea ésta. A fin de conocer las respuestas de la biota en estas lomas y como parte de los programas de monitoreo biológico por la RIBEN, un equipo del Museo de Historia Natural de la Universidad Nacional Mayor de San Marcos, viene realizando el seguimiento de la vegetación y de moluscos terrestres, y lo que aquí presentamos se refiere a la respuesta de dos especies de caracoles ( $B$. conspersus y S. peruviana) a "El Niño 1997-98",

\section{MATERIAL Y MÉTODOS}

\section{Lugar de estudio}

Para el programa de monitoreo de moluscos terrestres se eligió las Lomas de Lachay (11 $\left.20^{\prime} \mathrm{LS}, 77^{\circ} 23^{\prime} \mathrm{LW}\right), 105 \mathrm{~km}$ al norte de Lima, que es la más representativa de las lomas de la costa central del Perú (Saito, 1976) y es la única protegida por el Estado, como Reserva Nacional.

\section{Clima}

Las lomas tienen un clima estacional, producto de las neblinas advectivas que durante el invierno proveen de alta humedad. La "época húmeda", cuyo pico va de fines de julio a mediados de setiembre, se caracteriza por una alta humedad relativa, temperatura media inferior a $15^{\circ} \mathrm{C}$ y lloviznas frecuentes, que favorecen la vegetación de "lomas". Por el contrario, en el verano hay menor precipitación, baja humedad relativa (79 a $82 \%)$, y se presentan las mayores temperaturas medias mensuales $\left(20^{\circ} \mathrm{C}\right)$, originando una alta evapotranspiración, por lo que se conoce como "época seca", con un pico que va de diciembre a abril (Saito, 1976; Ordoñez y Faustino, 1983; Torres, 1985). Los eventos "El Niño" modifican esta estacionalidad, principalmente por las "garúas de verano", favoreciendo un período de "lomas" en el verano (Torres op. cit.; Pinche, 1994).

\section{Formaciones vegetales}

La vegetación de lomas está formada por especies herbáceas que reverdecen durante la "época húmeda", por lo que también se denomina "época de lomas", y especies perennes (arbustos y árboles) adaptadas también a la estacionalidad de las lomas. La distribución de la vegetación no es uniforme, sino que forma agrupaciones, por lo que los hábitats que presentan han sido denominados, según su predominancia, como "lomas de herbáceas", "lomas arbustivas", "lomas de bromeliáceas" y "lomas de cactáceas" (Ferreyra, 1953; Ono, 1986). En las Lomas de Lachay también hay especies introducidas, entre las que sobresalen Furcraea andina, Casuarina equisetifolia y Eucalyptus sp. Durante eventos "El Niño", la vegetación herbácea se expande considerablemente, siendo los porcentajes de cobertura vegetal reiterada (método del punto cuadrado) muy altos en pleno verano (Torres, op. cit.; Ono, op. cit.) como se pudo apreciar durante "El Niño 1997-98" (Cano et al., en este volumen).

\section{Especies}

Los caracoles terrestres $B$. conspersus (Bulimulidae) y $S$. peruviana (Succineidae) son especies que tienen una amplia distribución en las lomas, lo que les da una buena probabilidad de ser utilizadas como especies indicadoras.

B. conspersus (Sowerby, 1933) es la de mayor distribución, habiendo sido registrada en las lomas del centro y sur del país (Weyrauch, 1967; Aguilar y Arrarte, 1974). Es la especie dominante en las lomas de la costa central. En Lachay es encontrada en todos los hábitats terrestres, desde los 300 a $700 \mathrm{msnm}$. Durante la "época seca" estiva mayormente enterrada en los alrededores de la vegetación perenne y rocas, aunque puede 
ser encontrada en los resquicios de las rocas. Durante el período de actividad también suele enterrarse, sobre todo cuando las condiciones de humedad y temperatura se vuelven desfavorables, pero no forma epifragma resistente (Pulido y Ramírez, 1982; Ramírez, 1984; 1988).

La coloración de la conchilla es muy variada, puede ser agrupada en tres morfos: (1) castaño claro con manchas redondeadas blancas, (2) castaño intenso con manchas puntiformes a lineales, muy finas, y (3) castaño uniforme. El morfo (1) suele ser común (Ramírez, 1988).

S. peraviana (Philippi, 1867) se distribuye en las lomas del centro (Aguilar y Arrarte, 1974; Aguilar, 1985). En Lachay se encuentra, preferentemente, entre los 400 y $600 \mathrm{msnm}$, en lugares húmedos. Estiva en la parte baja de arbustos y árboles, así como enterrada superficialmente en suelo con abundante humus. La coloración marrón verdosa de su conchilla, en el animal vivo, la hace difícil de ubicar cuando están en el suelo o en la parte baja de arbustos, sobre todo a individuos juveniles (Pulido y Ramírez, 1982; Ramírez, 1984).

\section{Zona de muestreo}

Se eligió un área con predominio de vegetación arbustiva (e.g., Ophryosporus peruvianus, Senecio spp., Trixis cacalioides, Croton spp.), entre las cuales se desarrolla vegetación herbácea anual (e.g., Loasa urens, Nicotiana paniculata, Urocarpidium peruvianum y Nolana humifusa). Aquí, se delimitó una franja-transecto de $20 \times 200 \mathrm{~m}$, conteniendo cuatro parcelas de $10 \times 10 \mathrm{~m}$, distanciadas $50 \mathrm{~m}$ unas de otras, todas ellas ubicadas a una altitud promedio de $500 \mathrm{~m}$.

\section{Monitoreo}

Dentro del plan de monitoreo de vegetación y moluscos de las Lomas de Lachay, el equipo del Museo de Historia Natural (UNMSM) viene realizando salidas mensuales de 1-2 días desde julio de 1995. Se colectó hasta diciembre en 1995; en 1996 todo el año (excepto julio); en 1997, en marzo, mayo, julio-diciembre; y en 1998, hasta setiembre.

En la franja transecto fueron evaluados sólo los caracoles que estaban en la superficie, no así los que permanecían enterrados. La búsqueda de caracoles se hizo por observación directa; a cada caracol contado se medía la longitud de la conchilla ( $\mathrm{mm})$, y se tomaba datos de su comportamiento y relación al sustrato.

La densidad que se utiliza en las figuras corresponde al número de individuos encontrados en las cuatro parcelas juntas (ind. $\times 400 \mathrm{~m}^{-2}$ ). Para el análisis de la estructura poblacional, los caracoles fueron agrupados por tallas con intervalos de $5 \mathrm{~mm}$. Los datos fueron procesados en Microsoft Excel.

\section{RESULTADOS}

La actividad estacional de $B$. conspersus y S. peruviana en las Lomas de Lachay, fue analizada desde julio de 1995 hasta setiembre de 1998. Se observó la variación estacional de la actividad de los caracoles hasta 1997, aunque con diferente intensidad. En el verano de 1998 se rompió este esquema (Fig. 1).

\section{Respuesta de Bostryx conspersus a "El Niño 1997-98"}

\section{- Actividad}

La actividad de $B$. conspersus mostró clara evidencia de estacionalidad de 1995 a 1997. En 1995 el pico de actividad estuvo entre setiembre y diciembre. En 1996 el pico estuvo entre mayo y setiembre, decayendo hacia diciembre. El verano de 1997 fue particularmente adverso, no se avistó caracoles estivando en superficie; el pico de actividad se retrasó y se acortó a tres meses (agosto octubre), comparado con 1996; luego la actividad decayó, pero no se notó una clara evidencia de estivación. En enero de 1998, $B$. 


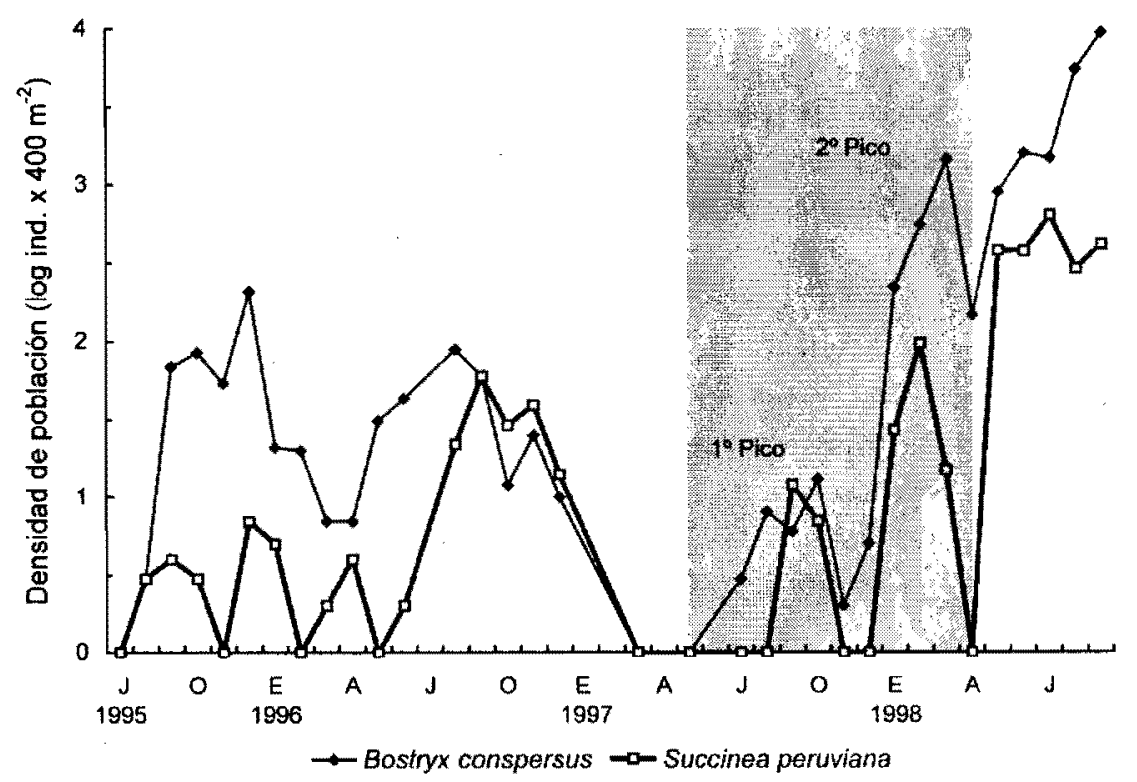

Figura 1. Variación de la densidad de poblaciones de $B$. conspersus y $S$. peruviana en las Lomas de Lachay, desde julio de 1995 a setiembre de 1998 (área sombreada: "El Niño 1997-98").

conspersus se encontraba activa y no cesó hasta lo que va del año (setiembre de 1998).

Con respecto a las máximas densidades alcanzadas por $B$. conspersus, se tiene que 1996 fue ligeramente menor que los máximos alcanzados en 1995. Hubo una caída estrepitosa de la población en 1997. Contrariamente, en 1998 hubo una explosión de la población, con dos picos, uno en marzo (más de cinco veces a lo alcanzado en diciembre de 1995), y otro en setiembre, con una densidad de más de seis veces a la alcanzada en el primer pico de 1998 (Fig. 1).

\section{- Reclutamiento}

Durante los años 1995 y 1996 el reclutamiento parece haber sido adecuado, en el pico de su actividad hubo mayores densidades de las primeras tallas que de adultos. En 1997, el reclutamiento estuvo claramente disminuido. En 19.98 el reclutamiento se incrementó considerablemente, no sólo con respecto a 1997 sino a los años anteriores, inclusive empezando en el verano.

La composición por tallas de $B$. conspersus en los años 1995 y 1996 fue constante, presentándose las cuatro clases de tamaños de una manera relativamente semejante. En 1997, ni durante el pico de actividad hubo individuos de las cuatro tallas en las proporciones de años anteriores. En 1998, se notó dos composiciones diferenciadas, para el verano una proporción de las dos tallas menores y la de adultos semejante a la de 1995, con la diferencia que la proporción del tercer tamaño $(10,1$ - $15 \mathrm{~mm})$ estuvo tremendamente incrementada. En el segundo pico de 1998 se rompió el esquema, las tres primeras tallas siguieron en ascenso, mientras que contrariamente, los individuos de mayor talla (>15 mm) disminuyeron (Fig. 2).

\section{Respuesta de Succinea peruviana a "El Niño 1997-98"}

\section{- Actividad}

La actividad estacional de S. peruviana fue evidente en 1996, alcanzando su pico entre agosto y diciembre. En 1997, estuvieron activos sólo dos meses (setiembre - octubre). En el verano de 1998 tuvieron un pico de actividad (enero - marzo), decayendo en abril. 


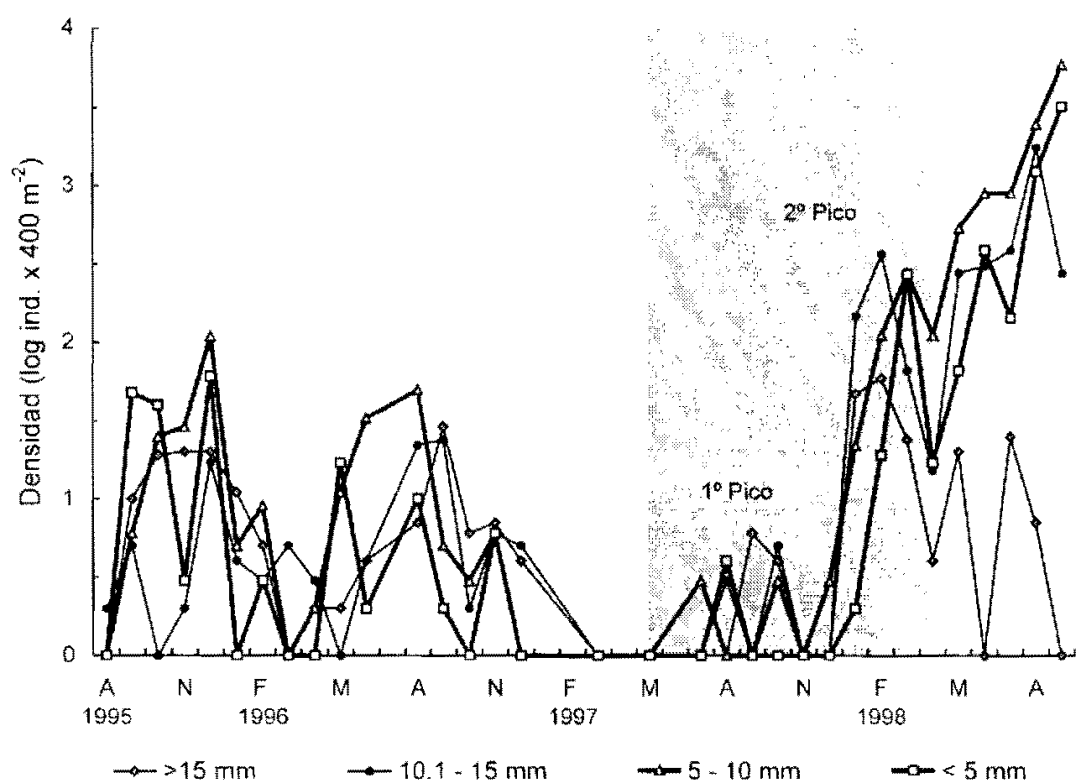

Figura 2. Estructura por tallas de la población de B. conspersus desde agosto 1995 hasta setiembre 1998 en las Lomas de Lachay (área sombreada: "El Niño 1997-98").

En adelante volvió a observarse otro pico de actividad, que supera al máximo del verano, manteniéndose hasta lo que va del año (setiembre de 1998).

Respecto a las densidades alcanzadas, pasó de ser rara en 1995 (menos de 10 individuos en $400 \mathrm{~m}^{2}$ ) a 60 ind. $\times 400 \mathrm{~m}^{-2}$ en el pico de actividad de 1996. En el corto período de actividad de 1997 las densidades volvieron a ser muy bajas, sólo ligeramente superiores a las de 1995. En 1998 hubo también una explosión poblacional, aunque menor que la de $B$. conspersus, alcanzó cerca de 100 ind. $\times 400 \mathrm{~m}^{-2}$ en el verano (febrero) y su máximo (632 ind. $\times 400 \mathrm{~m}^{*}$ ) durante el invierno (julio) (Fig. 1).

\section{- Reclutamiento}

La composición de la población por tallas fue muy errática durante los años 1995-1997. y definitivamente el reclutamiento estuvo muy disminuido. Al inicio de la temporada de actividad en 1996 fueron observados individuos de las primeras tallas $(<5-10 \mathrm{~mm})$ y en el pico de la "época húmeda" se tenía las tres tallas mayores, con la presencia de adultos "grandes" (15,1 - $20 \mathrm{~mm})$. En la "época húmeda" de 1997 nuevamente el reclutamiento fue infimo. En el verano de 1998, el reclutamiento no fue importante, como si lo fue después de abril. Los adultos con conchilla de longitud superior a $15 \mathrm{~mm}$ siguieron siendo muy raros (Fig. 3).

\section{Relación entre la cobertura vegetal reiterada y las densidades mensuales de B. conspersus y S. peruviana}

En relación a la respuesta de la vegetación herbácea y la de los moluscos a "EI Niño 199798" y el post-Niño, que en buena cuenta están respondiendo a la variación de la humedad y precipitación, no son las mismas o de igual intensidad. Al analizar la relación entre las densidades mensuales de $B$. conspersus y las de la cobertura vegetal reiterada (Fig. 4), se notó que durante el primer pico no fueron en ascenso proporcional, aunque el inicio de la "época de lomas" se retrasó en ambos casos (agosto $97=\mathrm{A} 7$ ), la cobertura vegetal alcanzó niveles que solía tener. Con respecto al 


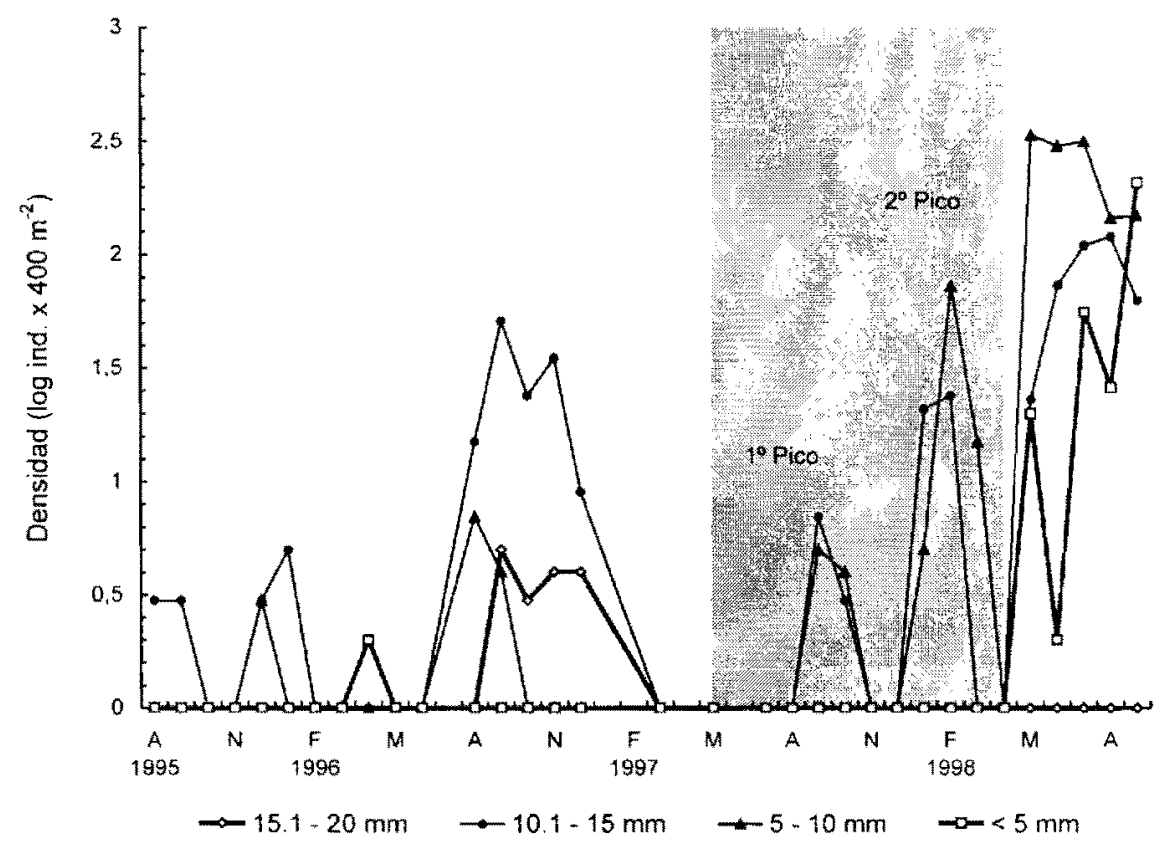

Figura 3. Estructura por tallas de la población de S. peruviana desde agosto de 1995 hasta setiembre de 1998 en las Lomas de Lachay (área sombreada: "El Niño 1997-98").

segundo pico, aquí sí se aprecia una relación positiva, como también durante el post-Niño.

En el caso de S. peruviana la relación (Fig. 5) tiene la misma tendencia que la mostrada por $B$. conspersus con la diferencia que sus densidades no alcanzaron proporciones extraordinarias.

\section{DISCUSIÓN}

El seguimiento de la variación de las densidades mensuales de $B$. conspersus y $S$.

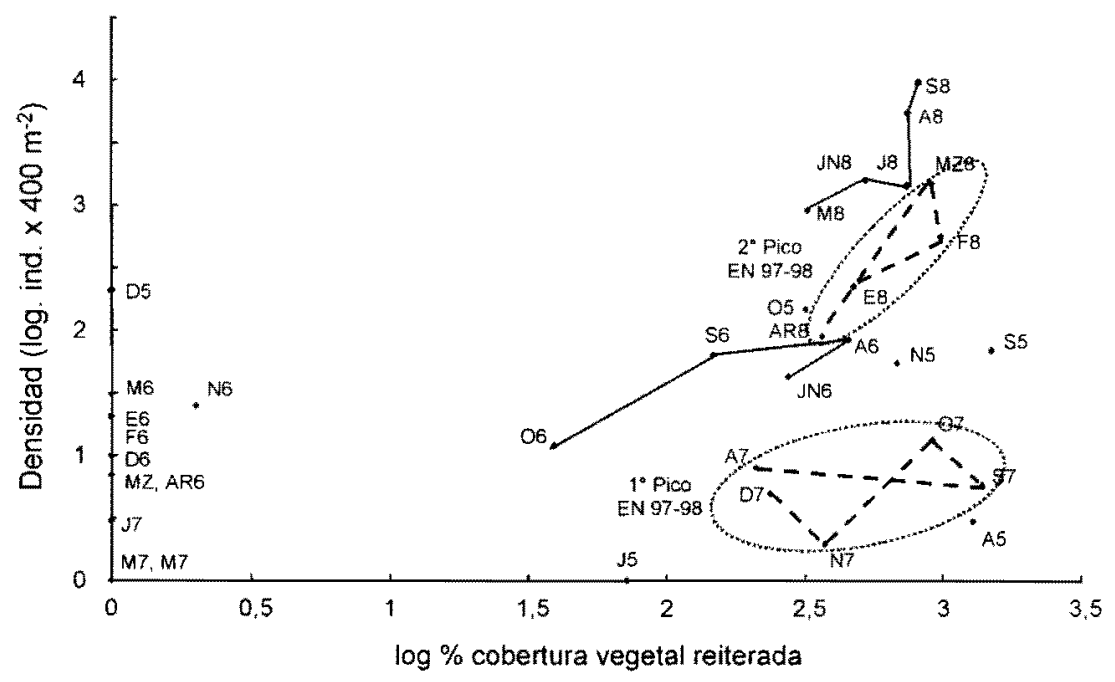

Figura 4. Relación entre la densidad de $B$. conspersus y la cobertura vegetal reiterada desde julio de 1995 hasta setiembre de 1998 en las Lomas de Lachay. 


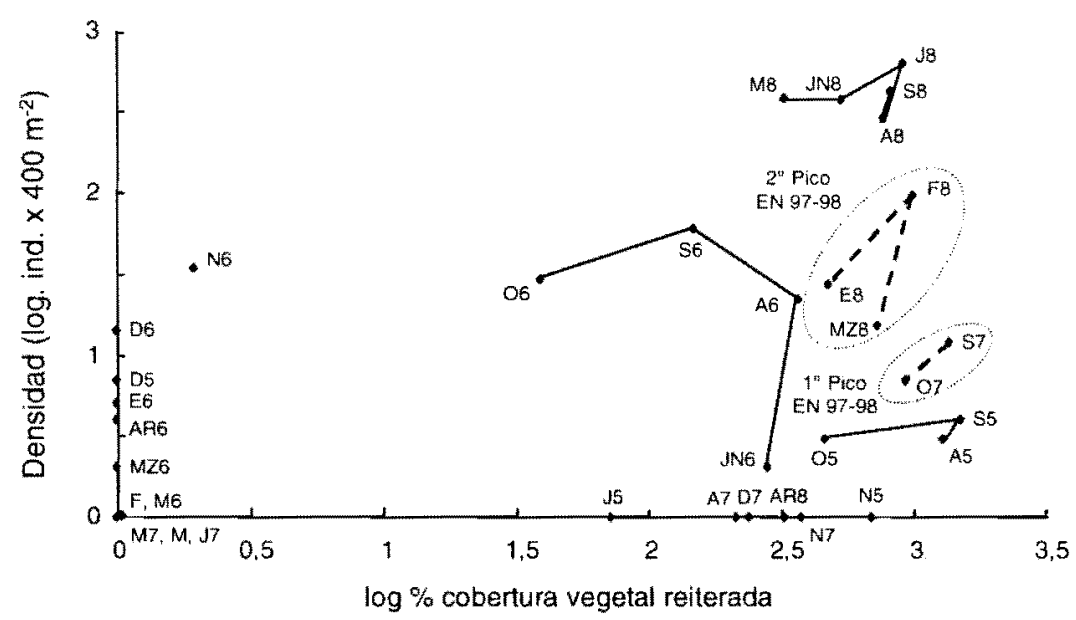

Figura 5. Relación entre la densidad de $S$. peruviana y la cobertura vegetal reiterada desde julio de 1995 hasta setiembre de 1998 en las Lomas de Lachay.

peruviana (número de individuos en superficie) de las lomas de Lachay permitió evaluar su comportamiento antes, durante y después de "El Niño 1997-98". Climáticamente, 1996 fue un año frío; "El Niño" estuvo presente entre mayo de 1997 y abril de 1998, con dos picos de calentamiento, otoño de 1997 y verano de 1998 (Ramos et $a l$, en este volumen; Tarazona et al., en este volumen), lo cual redundó en la vegetación, retrasando el inicio y prolongando la "época de lomas" hasta 1998 (Agüero y Suni, en este volumen). La respuesta a todos estos cambios climáticos por parte de $B$. conspersus y $S$. peruviana fue diferencial.

Durante el año frío (1996) B. conspersus tuvo un inicio temprano en su actividad (mayo), mientras que en la primera fase de "El Niño 1997-98" hubo un inicio tardío, postergándose a julio de $1997 ;$ S. peruviana "despertó" más tarde que $B$. conspersus en ambos años. Adicionalmente, la primera fase de "El Niño 1997-98" tuvo un efecto negativo en las poblaciones de $B$. conspersus y $S$. peruviana, pues no permitió el reclutamiento adecuado.

El segundo pico de "El Niño 1997-98", en el verano de 1998, por el contrario, tanto la especie dominante en el pre-Niño $(B$. conspersus) como a la que fue rara ( $S$. peruviana), tuvieron un pico de actividad, en una época que suelen estivar. Con ello se activó la maquinaria reproductiva, y como las condiciones de humedad y cobertura vegetal eran óptimas, la supervivencia fue mayor, y no murieron muchos individuos por desecación o falta de refugio (Figs. 4 y 5), principales controladores de las poblaciones de moluscos terrestres (Pollard, 1975; Ramírez, 1984). Una situación similar se dio con $B$. conspersus en las Lomas de Iguanil (Lima) en "El Niño" extraordinario de 1982-83 (Ramírez, op. cit.).

La prolongación de la "época de lomas" al verano no significa que se dan las mismas condiciones metereológicas; hay humedad y precipitación elevadas pero la temperatura es la de una "época seca" (Torres, 1985). Todas las especies tienen una diversidad genética que les confiere la posibilidad de adaptación ante las condiciones cambiantes de su medio, por lo que en una población saludable no todos los individuos son iguales (Primack, 1993). Esta diversidad puede ser apreciada fenotípicamente en $B$. conspersus, cuyos individuos con conchilla del morfo común (1) (Ramírez, 1988) fueron desplazados durante "El Niño 1997-98" por aquellos con conchillas 
de apariencia más bien rayada. En el caso de $S$. peruviana no es posible evidenciar esta diversidad genética externamente. Sin embargo, con buena probabilidad se podría identificar múltiples alelos en relación a enzimas involucrados en su fisiología, que los han hecho resistentes a condiciones no precisamente hidrófilas (las lomas son ecosistemas de desierto), y responden exitosamente a condiciones de alta humedad presentes durante la segunda fase de "El Niño 1997-98", como es común en otros representantes de la familia Succineidae que suelen vivir en lugares permanentemente húmedos (e.g., pantanos y márgenes de ríos y lagos) (Kerney y Cameron, 1979).

Durante el post-Niño nuevamente hubo un ascenso en la densidad de las poblaciones. Ello respondería a las condiciones climáticas propias de una "época de lomas", y que empiezan esta "nueva época húmeda" una mayor cantidad de individuos reproductores.

El descenso de adultos de $B$. conspersus en esta temporada parece haberse debido prioritariamente a la depredación por roedores, pues era muy común encontrar conchillas frescas y destruidas, (quedando casi siempre sólo la vuelta corporal) en la base de arbustos, en mayor proporción de lo observado en años no-Niño (Ramírez et al., 1997).

Otros miembros de la biota fueron afectados en similar forma por "El Niño 1997-98" en las Lomas de Lachay. Por ejemplo, Ismene amancaes ("amancaes") tuvo un inicio tardío y acortamiento de su desarrollo en 1997 (Agüero y Suni, op. cit.), y los árboles extendieron su período de floración "durante 1997-98, manteniendo individuos con flor casi todo un año, aunque con diferentes picos de abundancia" (Arana et al., 1998).

Agradecimientos: A la Organización de los Estados Americanos (por intermedio de la RIBEN), la Univ. Nac. Mayor de San Marcos (FEDU) y el CONCYTEC (Perú). Nuestro agradecimiento a José Arenas, Sergio Cano,
Doris Florindez, Ruth Miranda, Marisa Ocrospoma, Niel Salazar y María Samamé, por su apoyo entusiasta en el trabajo de campo.

\section{LITERATURA CITADA}

Aguilar. P. 1985. Fauna de las lomas costeras del Perú. Bol. Lima (41): 17-28.

. 1990. Sinopsis sobre los eventos del fenómeno "El Niño" en el Perú. Bol. Lima (70): 69-84

Aguilar, P. y J. Arrarte. 1974. Moluscos de las lomas costeras del Perú. Anales Científicos UNALM 12 (3-4): 93-98.

Arana, C; A. Cano; J. Roque; M. Arakaki; M. La Torre; N. Refulio y R. Ramírez. 1998. Respuesta de la vegetación leñosa de las Lomas de Lachay al evento “El Niño 1997-98”. IV Congreso Latinoamericano de Ecología. II Congreso Peruano de Ecología, 20-25 de octubre. Arequipa. Libro de Resúmenes, p. E-4.

Arntz, W. y E. Fahtbach. 1991. El Niño: Experimento climático de la naturaleza. Causas físicas y efectos biológicos. Fondo de Cultura Económica, México, 312 pp.

Arntz, W.; A. Landa y J. Tarazona (eds.). 1985. "El Niño", su impacto en la fauna marina. Bol. Inst. Mar Perú-Callao Vol. Extraor., 222 pp.

Bayona, R. 1985. El fenómeno "El Niño". En: M. Vega (ed.). Ciencia. Tecnología y Agresión Ambiental: El Fenómeno "El Niño". CONCYTEC. Lima. pp. 13-27.

Ferreyra, R. 1953. Comunidades vegetales de algunas lomas costaneras del Perú. Boletín Estación Experimental Agraria La Molina 53: 1-88.

Kerney, M. \& R. Cameron. 1979. A field guide to the land snails of Britain and North-west Europe. Collins, London, $288 \mathrm{pp}$.

Ono, M. 1986. Delinition, classification and taxonomic significance of the Lomas vegetation. $/ n$ : M. Ono (ed.). Taxonomic and Ecological Studies on the Lomas Vegetation in the Pacific Coast of Peru. Reports for Overseas Scientific Survey. Makino Herbarium. Tokyo Metropolitan University, pp. 5-14.

Ordoñez, J. y J. Faustino. 1983. Evaluación del potencial hídrico en lomas costeras del Perú (Lomas de Lachay - Iguanil). Zonas Áridas (3): 29-42.

Pinche, C. 1994. Estudio de las condiciones climáticas y de la niebla en la costa norte de Lima. Bol. Lima 16 (91-96): 39-43.

Pollard, E. 1975. Aspects of the ecology of Helix pomatia L. J. Anim. Ecol. 44 (1): 305-329.

Primack, R. 1993. Essentials of conservation biology. Sinauer Associates Inc. Sunderland. Massachusetts, USA, $564 \mathrm{pp}$.

Pulido, V. y R. Ramírez. 1982. Distribución y actividad 
estacional de los caracoles terrestres de las Lomas de Lachay. VII Congreso Nacional de Biología y II Simposium de Educación en Ciencias Biológicas, 22-27 noviembre, Lima, Perú, Bitácora Biológica. Libro de Resímenes, I (1): 52 .

Ramírez, R. 1984. Aspectos de la ecología de Bostryx conspersus (Sowerby. 1833) (Mollusca, Bulimulidae) en las Lomas de Iguanil, Huaral-Lima. Informe de Práticas Pre-Profesionales para optar el Título Profesional de Biólogo. Universidad Nacional Mayor de San Marcos, Lima, Perú, 63 pp. 1988. Morfología y biología de Bostryx conspersur (Sowerby) (Mollusca, Bulimulidae) en las lomas costeras del Perú central. Rev. Bras. Zool. 5 (4): 609-617.

Ramírez, R.: K. Caro y S. Córdova. 1997. Depredación de moluscos terrestres en las Lomas de Lachay. VI Reunión Científica, Inst. Invest. Ciencias Biológicas "Antonio Raimondi", 9-11 abril, Lima. Perü. Libro de Resúmenes. p. 103.
Rundel, P.W.; M.O. Dillon; B. Palma: H.A. Mooney; S.L. Gulmon \& J.R. Ehleringer. 1991. The phytogeography and ecology of the coastat Atacama and Peruvian deserts. Alixo 13 (1): 1-49.

Saito, C. 1976. Bases para el establecimiento y manejo de una unidad de conservación en las Lomas de Lachar, Peri. Ministerio de Agricultura. Dirección General Forestal y de Fauna. Dirección de Conservación. 205 pp.

Torres, J. 1985. Anomalias observadas en la vegetación y sus factores físicos determinantes en las Lomas de la Costa Central, durante el verano (enero-abril) de 1983. En: M. Vega (ed.) Ciencia, Tecnología y Agresión Ambiental: El Fenómeno "El Niño". CONCYTEC. Lima. pp. 125-642.

Vega, M. (ed.). 1985. Ciencia, Tecnologia y Agresión Ambiental: El Fenómeno "El Niño". CONCYTEC Lima, Perú. 692 pp.

Weyrauch. W. 1967. Treinta y ocho nuevos gasterópodos terrestres del Perú. Acta Zoologica Lilloana 21 : 343-455. 